\title{
THE METHOD OF NONFLAT TIME EVOLUTION (MONTE) IN PDE-BASED IMAGE RESTORATION
}

\author{
Youngjoon Cha*, Seongjai Kim
}

\begin{abstract}
This article is concerned with effective numerical techniques for partial differential equation (PDE)-based image restoration. Numerical realizations of most PDE-based denoising models show a common drawback: loss of fine structures. In order to overcome the drawback, the article introduces a new time-stepping procedure, called the method of nonflat time evolution (MONTE), in which the timestep size is determined based on local image characteristics such as the curvature or the diffusion magnitude. The MONTE provides PDE-based restoration models with an effective mechanism for the equalization of the net diffusion over a wide range of image frequency components. It can be easily applied to diverse evolutionary PDE-based restoration models and their spatial and temporal discretizations. It has been numerically verified that the MONTE results in a significant reduction in numerical dissipation and preserves fine structures such as edges and textures satisfactorily, while it removes the noise with an improved efficiency. Various numerical results are shown to confirm the claim.
\end{abstract}

Key Words : Method of nonflat time evolution (MONTE), net diffusion (ND) function, diffusion equalization, fine structures, total variation (TV) model, numerical dissipation

\section{I . INTRODUCTION}

Image restoration is an important image processing (IP) step for various image-related applications and is often necessary as a pre-processing for other imaging techniques such as segmentation, registration, and compression and visualization. Thus image restoration methods have been considered as an important process in IP, computer graphics, and their applications ${ }^{[1,2,3,4]}$.

There have been lots of partial differential equation (PDE)-based models in image restoration such as the Perona-Malik model ${ }^{[5]}$, the total variation (TV) minimization ${ }^{[6,7]}$, and color restoration models ${ }^{[8,}$ 9,10]. These PDE-based models have been extensively studied to answer fundamental questions in image restoration and have allowed researchers and practitioners not only to introduce new mathematical models but also to analyze and improve traditional algorithms ${ }^{[11,12,13]}$. Good references to work on them are e.g. AubertKornprobst $^{[14]}$, Osher-Fedkiw ${ }^{[15]}$, and Sapiro ${ }^{[4]}$.

However, most of these PDE-based restoration models and their numerical realizations show a common drawback: loss of fine structures. In particular, they often introduce an excessive and undesirable numerical dissipation on regions where the image content changes rapidly such as on edges and textures. Therefore it is very important and challenging to develop mathematical models and numerical techniques which can effectively preserve fine structures during the restoration. Although there have been developed new mathematical models for a better preservation of fine structures ${ }^{[2,16]}$, more

※ The work of the first author was supported by the National Research Foundation of Korea(NRF) grant funded by the Korea government(MEST) (No. 20110179).

- 주저자 : Department of Applied Mathematics, Sejong University, Gunja-Dong 98, Seoul 143-747, Korea, yjcha@sejong.ac.kr, 정회원

* Department of Mathematics and Statistics, Mississippi State University, Mississippi State, MS 39762-5921, USA, skim@math.msstate.edu

논문번호 : KICS2012-09-459, 접수일자 : 2012년 9월 26일, 최종논문접수일자 : 2012년 11월 8일 
advanced strategies have yet to be developed.

In order to effectively suppress the numerical dissipation, this article employs a new time-stepping procedure, called the method of nonflat time evolution (MONTE), in which the timestep size is determined locally depending on image characteristics such as the curvature and the diffusion magnitude. As observed in Section 3 below, diffusion terms in PDE-based restoration models tend to impose an extravagant dose of diffusion near fine structures, which in turn introduces a higher level of numerical dissipation there. The MONTE controls the timestep size $\Delta t$ to equalize the net diffusion over a wide range of image frequency components; the MONTE sets $\Delta t$ smaller on the regions where the diffusion magnitude is larger. Thus the MONTE can be viewed as a diffusion equalization technique. This new strategy evolves the pseudo-time through nonflat surfaces and is able to significantly reduce numerical dissipation particularly near fine structures.

An outline of the paper is as follows. In the next section, we first introduce a general denoising model representing diverse PDE-based restoration models and then present a brief review of the total variation (TV)-based models, followed by an efficient numerical algorithm for the general denoising model. Section 3 discusses the MONTE in detail. In the same section, the net diffusion (ND) function is defined to represent the total diffusion resulted from the application of the MONTE. Section 4 shows the stability of the MONTE using the maximum principle; it does not introduce local extrema having values outside the range of the noisy image. Section 5 contains numerical experiments for the MONTE. Four models are compared for their effectiveness (in terms of visual verification and PSNR analysis) and efficiency, for various synthetic and real images. The MONTE results in a significant reduction in numerical dissipation for all images we have tested. The last section concludes our development and experiments.

\section{PRELIMINARIES}

This section begins with the introduction of a general denoising model which represents various popular restoration models. Then, we present a brief review of the TV-based models, followed by an efficient numerical algorithm for the general model.

\subsection{A general denoising model}

Let $f$ be the observed image of the form

$$
f=u+v,
$$

where $u$ is a desired image and $v$ denotes noise or the residual. Then, most PDE-based image denoising models can be formulated in their evolutionary form as

$$
\frac{\partial u}{\partial t}+L u=R(f-u)
$$

where $L$ is a nonlinear diffusion operator and $R$ denotes a nonnegative constraint term. For example, the Perona-Malik (PM) $\operatorname{model}^{[5]}$, the TV model ${ }^{[7]}$, the improved total variation (ITV) $\operatorname{model}^{[6]}$, and the $\alpha \beta \omega(\mathrm{ABO})$ model $^{[9]}$ can be expressed as (2) with the following choices:

(a) $L u=-\nabla \cdot(g(|\nabla u|) \nabla u), R=0, \quad(\mathrm{PM})$

(b) $L u=-\kappa_{1}(u), R=\lambda$,

(c) $L u=-|\nabla u| \kappa_{1}(u), R=\lambda|\nabla u|$,

(d) $L u=-|\nabla u|^{q} \kappa_{q}(u), R=\beta(\mathrm{x}, t)$,

where $\lambda>0, \alpha \geq 0, \omega \geq 1$ are constants and $\kappa_{q}$ and $g$ are functions of the form

$$
\begin{array}{ll}
\kappa_{q}(u)=\nabla \cdot\left(\frac{\nabla u}{|\nabla u|^{q}}\right), & q \geq 0, \\
g(s)=\frac{1}{1+s^{2} / \sigma^{2}}, & \sigma>0 .
\end{array}
$$

Here $\beta$ is a nonnegative function of $f$ and $u$; see [9] for an automatic and yet effective strategy of choosing a variable constraint parameter. The ABO model has been applied as an effective edge-forming method for image zooming which incorporates large and non-integer magnification factors ${ }^{[17,18,19]}$. 
In the following, we present a brief review of the TV-based models, as examples of the general model (2).

\subsection{The TV-based models}

A common denoising technique is to minimize a functional of gradient, given as

$$
u=\arg \min _{u} F_{p}(u)
$$

where

$$
F_{p}(u)=\int_{\Omega}|\nabla u|^{p} d \mathrm{x}+\frac{\lambda}{2}\|f-u\|^{2} .
$$

Here $\lambda$ and $\|\cdot\|$ denotes the $L^{2}$-norm. When $p=1$, the first term in $F_{1}(u)$ is called the total variation (TV). By applying the variational calculus $^{[20]}$, one can transform the minimization problem (5) into an equivalent differential equation, called the Euler-Lagrange equation:

$$
-\nabla \cdot\left(\frac{\nabla u}{|\nabla u|^{2-p}}\right)-\frac{\lambda}{p}(f-u)=0 .
$$

For a convenient numerical simulation of (6), we parametrize the energy descent direction by an artificial time $t$; the resulting evolutionary Euler-Lagrange equation can be formulated as:

$$
\frac{\partial u}{\partial t}-\nabla \cdot\left(\frac{\nabla u}{|\nabla u|^{2-p}}\right)=\frac{\lambda}{p}(f-u)
$$

Note that the restored image becomes closer to $f$ as $\lambda$ grows. We adopt the no-flux boundary condition, for simplicity, hereafter; the initial value of $u$ is $f$.

An interesting case in (7) is when $p=1$, the $T V$ model:

$$
\frac{\partial u}{\partial t}-\nabla \cdot\left(\frac{\nabla u}{|\nabla u|}\right)=\frac{\lambda}{p}(f-u) .
$$

Although the TV model pertains certain attractive mathematical properties in image restoration, its numerical realization introduces a large numerical dissipation near fine structures. It is also known that the TV model tends to transform the image into a collection of locally constant portions, which is called the staircasing. The removal of staircasing has been an interesting research topic. As an anti-staircasing approach, Marquina and Osher ${ }^{[6]}$ introduced the improved total variation (ITV) model

$$
\frac{\partial u}{\partial t}-|\nabla u| \nabla \cdot\left(\frac{\nabla u}{|\nabla u|}\right)=\lambda|\nabla u|(f-u) .
$$

The above model can be derived by scaling the stationary TV model, (6) with $p=1$, by a factor of $|\nabla u|$ and then introducing the time parametrization. The scaling can suppress the staircasing effectively, as indicated in [15]; for its ability to reduce numerical dissipation, see Remark 1 in Section 3 below.

\subsection{The numerical algorithm}

For a numerical discretization, we let $\Delta t$ be a prescribed timestep size and let $t^{n}=n \Delta t$ and $u^{n}=u\left(\cdot, t^{n}\right)$. Then, an incomplete (linearized) Crank-Nicolson scheme for the general model (2) reads

$$
\frac{u^{n}-u^{n-1}}{\Delta t}+B^{n-1} \frac{u^{n}+u^{n-1}}{2}=R^{n-1} f
$$

where $B^{n-1}$ is the diffusion matrix which can be defined depending on the selected model. For example, for the ABO model, one can set

$$
B_{l}^{n-1} u^{m}=A_{l}^{n-1} u^{m}+\frac{R^{n-1}}{2} u^{m},
$$

and

$$
A_{l}^{n-1} u^{m}:=-\mid \nabla_{h} u^{n-1 \mid \alpha} D_{x_{l}}\left(\frac{D_{x_{l}} u^{m}}{\mid \nabla_{h} u^{n-1 \mid \omega}}\right)
$$

for $\quad l=1,2 \quad$ and $\quad m=n, n-1$. Here $\left(D_{x_{1}}, D_{x_{2}}\right)^{T}$ is the half-step central difference operator for the gradient $\nabla$ and $\left|\nabla_{h} u^{n-1}\right|$ denotes a numerical approximation of gradient magnitude $|\nabla u|$ such as the (standard) second-order central scheme. The linear system (10) may be solved by directly applying an iterative algebraic solver. However, it can be perturbed in order to apply the alternating direction implicit (ADI) time-stepping procedure ${ }^{[21}$, 22]. 


$$
\begin{aligned}
&\left(1+\frac{\Delta t}{2} B_{1}^{n-1}\right) u^{*}=\left(1-\frac{\Delta t}{2} B_{1}^{n-1}-\Delta t B_{2}^{n-1}\right) u^{n-1} \\
&+\Delta t R^{n-1} f \\
&\left(1+\frac{\Delta t}{2} B_{2}^{n-1}\right) u^{n}=u^{*}+\frac{\Delta t}{2} B_{2}^{n-1} u^{n-1} .
\end{aligned}
$$

where $u^{*}$ is an intermediate solution.

In this article we will call (13) the Crank-Nicolson ADI (CN-ADI) algorithm.

\section{The METHOD OF NONFLAT TIME EVOLUTION (MONTE)}

In this section, we will introduce the MONTE, as a time-stepping procedure, for the general model (2). We begin with an observation for sources of numerical dissipation.

\subsection{An observation}

For simplicity, we momentarily assume that the given image $f$ involves no noise such that the desired image $u$ is the same as $f$, i.e., $v^{0}=0$ and $u^{0}=f$. The assumption will help us understand why the PDE-based models result in more extensive numerical dissipation near fine structures.

Utilizing (1), the model (2) can be rewritten as

$$
\frac{\partial v}{\partial t}+R v=L u \text {. }
$$

The above equality holds also for the numerical residual at the $n$-th time level, $v^{n}\left(:=f-u^{n}\right)$, for all $n \geq 0$ :

$$
\frac{\partial v^{n}}{\partial t}+R^{n} v^{n}=L^{n} u^{n}
$$

Thus, since $v^{0}=0$, the $n$-th residual $v^{n}$, $n \geq 0$, remains zero in regions where, $L^{k} u^{k}=0$, $0 \leq k \leq n$, while it becomes nonzero otherwise.

Let us discuss it in detail with an example. Consider the residual equation for the TV model (8):

$$
\frac{\partial v^{n}}{\partial t}+\lambda v^{n}=-\kappa\left(u^{n}\right)
$$

Then, it is not difficult to see that for the TV model, the residual becomes positive or negative at pixels where the image is concave down or concave up, respectively. Thus the numerical solution $u^{n}$ of the TV model must involve numerical dissipation wherever its curvature is nonzero. Furthermore, we can reach the conclusion that the TV model (8) diffuses the image more actively as the local values of the curvature become larger in modulus.

The above conclusion for the TV model can be extended to general images and the general model (2) as well: the bigger the diffusion magnitude $\left|L^{n} u^{n}\right|$ is, the larger numerical dissipation the numerical solution $u^{n}$ incorporates.

\subsection{The MONTE time-stepping procedure}

The observation in the previous subsection has motivated us to develop the following strategy. In the simulation of the model (2) utilizing the CN-ADI (13), we try to control the amount of diffusion by setting the timestep size $\Delta t$ dynamically, depending on the diffusion magnitude for the last iterate $L^{n-1} u^{n-1}$. ((Note that $u^{n}$ is not available at the beginning of the $n$-th time level.) That is, we set $\Delta t^{n}=\Delta t\left(\cdot, t^{n}\right)$ as

$$
\Delta t\left(\mathrm{x}, t^{n}\right)=\Delta t_{0} \cdot F\left(L u\left(\mathrm{x}, t^{n-1}\right)\right),
$$

where $\Delta t_{0}$ is a constant and $F$ is a scaling function. For example, we may define $F$ as follows: for positive constants $\eta$ and $\gamma$,

$$
F(s)=\frac{\gamma}{1+\eta|s|} .
$$

Incorporating (16) into (13), the resulting algorithm reads

$$
\begin{aligned}
& \left(1+\frac{\Delta t^{n}}{2} B_{1}^{n-1}\right) u^{*} \\
= & \left(1-\frac{\Delta t^{n}}{2} B_{1}^{n-1}-\Delta t^{n} B_{2}^{n-1}\right) u^{n-1} \\
& +\Delta t^{n} R^{n-1} f, \\
& \left(1+\frac{\Delta t^{n}}{2} B_{2}^{n-1}\right) u^{n} \\
= & u^{*}+\frac{\Delta t^{n}}{2} B_{2}^{n-1} u^{n-1} .
\end{aligned}
$$




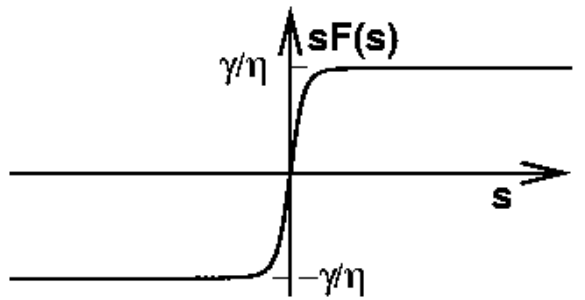

Fig. 1. The ND function $s F(s)$ for certain choices of $\eta$ and $\lambda$.

The numerical solution $u^{n}$ in (18) has values on different time levels; it is defined on a nonflat time surface. The algorithm seeks the solution through a nonflat time evolution, for which we call the strategy the method of nonflat time evolution (MONTE). Concepts of surface evolution can be found in various research areas; we refer the reader to [23] among others. We will call (18) the MONTE-incorporated $\quad C N-A D I \quad$ (M-CN-ADI) algorithm.

We close the section with a couple of remarks on the MONTE.

Remark 1. A standard numerical realization of the ITV model (9), [6] can be viewed as a MONTE applied for the TV model (8), with the choice

$$
\Delta t\left(\mathrm{x}, t^{n}\right)=\Delta t_{0} \cdot\left|\nabla_{h} u^{n-1}(\mathrm{x})\right|,
$$

Thus it can reduce staircasing effectively; however, it is hardly concerned with the reduction of numerical dissipation.

Remark 2. Along with the scaling function $F$ in (17), the M-CN-ADI (18) applied to (2) can be viewed as a numerical realization of the following model:

$$
\frac{\partial u}{\partial t}+\frac{\gamma}{1+\eta|L u|} L u=\frac{\gamma}{1+\eta|L u|} R(f-u) .
$$

Hence, it is clear to see that the resulting algorithm must incorporate a total diffusion of the form $s F(s), \quad s=L u$, as depicted in Figure 1 . Associated with (20), the residual equation becomes

$$
\frac{\partial v}{\partial t}+\frac{\gamma}{1+\eta|L u|} R v=\frac{\gamma}{1+\eta|L u|} L u .
$$

We call $s F(s)$ the net diffusion (ND) function in this article.
Remark 3. It can be verified that the diffusion magnitude $|L u|$ on regions of fine structures is larger than the average value of the diffusion magnitude over the whole domain. Let $L_{0}$ denote the average value of the diffusion magnitude. When the ratio $\frac{\gamma}{\eta}$ is selected as

$$
\frac{\gamma}{\eta}=\frac{L_{0}}{\xi}
$$

for some $0<\xi<1$, one can prove that

$$
L_{0} \leq|s F(s)| \leq L_{0} \cdot \frac{1}{\xi} \text {, for }|s| \geq L_{0} .
$$

Thus the net diffusion on oscillatory regions $\left(|L u| \geq L_{0}\right)$ can differ by a factor of $\frac{1}{\xi}$. The above inequality also implies that by selecting $\eta$ and $\gamma$ appropriately, one can impose diffusion equalization over a wide range of the diffusion magnitude and therefore the algorithm may hardly introduce excessive numerical dissipation near fine structures. See (29) below for a choice of $\eta$ and $\gamma$.

\section{STABILITY OF THE MONTE}

In this section, we presents a stability analysis for the MONTE; the conclusion shows that the new method do not introduce local extrema having values outside the range of the original image.

Theorem 4.1. Consider the general denoising model (2) with the numerical algorithm (10), (11) and (12) incorporated with the MONTE algorithm described in (16) and (17). Let $R_{0}=\sup R(\mathrm{x}, t)$, $F_{0}=\sup _{s} F(s)$, and the following condition is satisfied.

$$
\left(2+\frac{R_{0}}{2}\right) \Delta t_{0} F_{0} \leq 1
$$

Then

$$
\min _{i, j} f_{i j} \leq u_{i j}^{n} \leq\|f\|_{\infty}, n \geq 0 .
$$

where $u_{i j}^{n}$ is the approximate solution at the pixel $\mathrm{X}_{i j}$ on the time level $t^{n}$ and $\|f\|_{\infty}:=\max _{i j}\left|f_{i j}\right|$, the maximum norm of $f$. 


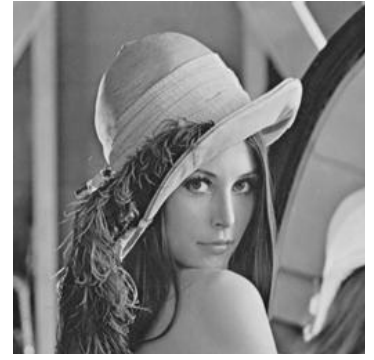

(a)

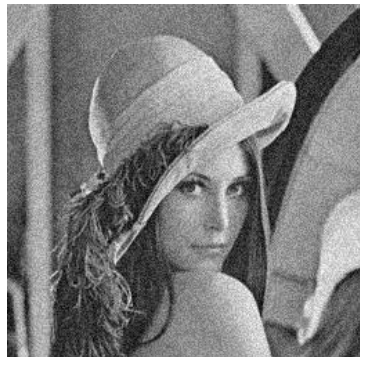

(b)
Fig. 2. Lenna: (a) The original image $g$ and (b) a noisy image $f$ contaminated by a Gaussian noise (PSNR $=24.8$ ).

Proof. Among the models listed in (3), let's consider the $\mathrm{ABO}$ model first. In that case, we may choose $1<\alpha=\omega<2$ and $R=\beta(\mathrm{x}, t)^{[9]}$, and then, use the following notation as in [18], i. e.,

$$
\begin{aligned}
& a_{i j, W}^{n-1}=\frac{2 d_{i j, E}^{n-1}}{d_{i j, W}^{n-1}+d_{i j, E}^{n-1}}, \\
& a_{i j, E}^{n-1}=\frac{2 d_{i j, W}^{n-1}}{d_{i j, W}^{n-1}+d_{i j, E}^{n-1}} .
\end{aligned}
$$

Here $d_{i j, W}^{n-1}$ and $d_{i j, E}^{n-1}$ can be determined as follows:

$$
\begin{aligned}
& d_{i j, W}^{n-1}=\left[\left(D u_{i-1 / 2 . j}^{n-1}\right)^{2}+\varepsilon^{2}\right]^{\omega / 2}, \\
& d_{i j, E}^{n-1}=d_{i+1, j, W}^{n-1},
\end{aligned}
$$

where $D u_{i-1 / 2, j}^{n-1} \quad$ is a finite difference approximation of $\left|\nabla u^{n-1}\right|$ evaluated at $\mathrm{x}_{i-1 / 2, j}$, the mid point of $\mathrm{X}_{i-1, j}$ and $\mathrm{X}_{i, j}$. Then, since $a_{i j, W}^{n-1}+a_{i j, E}^{n-1}+a_{i j, S}^{n-1}+a_{i j, N}^{n-1}=4$, the equation (11) at a point $\mathrm{X}_{i, j}$ can be written as

$$
\begin{aligned}
& {\left[1+\left(4+R_{i j}^{n-1}\right) \frac{\Delta t_{i j}^{n-1}}{2}\right] u_{i j}^{n}} \\
& =\frac{\Delta t_{i j}^{n-1}}{2}\left[a_{i j, W}^{n-1} u_{i-1, j}^{n}+a_{i j, E}^{n-1} u_{i+1, j}^{n}\right. \\
& \left.+a_{i j, S}^{n-1} u_{i, j-1}^{n}+a_{i j, N}^{n-1} u_{i, j+1}^{n}\right] \\
& +\frac{\Delta t_{i j}^{n-1}}{2}\left[a_{i j, W}^{n-1} u_{i-1, j}^{n-1}+a_{i j, E}^{n-1} u_{i+1, j}^{n-1}\right. \\
& \left.+a_{i j, S}^{n-1} u_{i, j-1}^{n-1}+a_{i j, N}^{n-1} u_{i, j+1}^{n-1}\right] \\
& +\left[1-\left(2+\frac{R_{i j}^{n-1}}{2}\right) \Delta t_{i j}^{n-1}\right] u_{i j}^{n-1} \\
& +\Delta t_{i j}^{n-1} R_{i j}^{n-1} f i j,
\end{aligned}
$$

where

$$
R_{i j}^{n-1}=R\left(\mathrm{x}_{i j}, t_{i j}^{n-1}\right)
$$

and

$$
t_{i j}^{n-1}=\Delta t\left(\mathrm{x}_{i j}, t^{n}\right)=\Delta t_{0} \cdot F\left(L u\left(\mathrm{x}_{i j}, t^{n-1}\right)\right) .
$$

Note that (27) is also true for all the models listed in (3), and hence we may say that (27) is true for general model (2). Then, it follows from (23) that each of coefficients in the right side of (26) is nonnegative and their sum becomes $1+\left(4+R_{i j}^{n-1}\right) \frac{\Delta t_{i j}^{n-1}}{2}$. Thus we have the desired result.

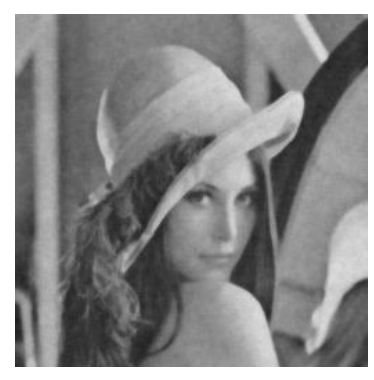

(a)

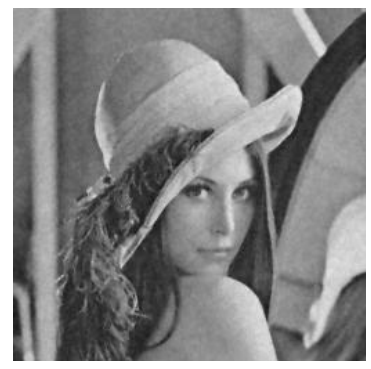

(c)

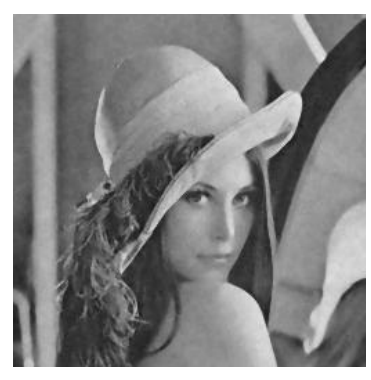

(b)

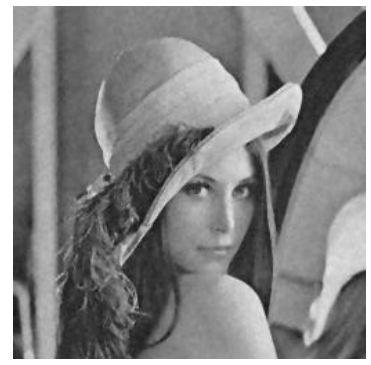

(d)
Fig. 3. Lenna: Restored images $u$ by (a) ITV, (b) ABO, (c) M-ITV, and (d) M-ABO. The noisy image is given in Figure 2(b). 


\section{NUMERICAL EXPERIMENTS}

In this section, we verify effectiveness of the MONTE applied to image denoising. Since the first three models in (3) can introduce a severe staircasing or numerical dissipation due to a weak stability or no constraint term, we select the last two in (3), ITV and ABO, as background models for the verification. We have implemented four different models: ITV, ABO, the ITV incorporating the MONTE (M-ITV), and the ABO incorporating the MONTE (M-ABO). For the ABO-based models (ABO and $\mathrm{M}-\mathrm{ABO}$ ), we set parameters $\alpha=\omega=1.8$. As a trial image, we choose Lenna in gray-scale, Figure 2(a), which is one of most popular trial images in IP. As shown in Figure 2(b), the image is perturbed by a Gaussian noise of $P S N R=24.8$. Here the PSNR (peak signal-to-noise ratio) is defined as

$$
\operatorname{PSNR} \equiv 10 \log _{10}\left(\frac{\sum_{i j} 255^{2}}{\sum_{i j}\left(f_{i j}-h_{i j}\right)^{2}}\right) \mathrm{dB},
$$

where $f$ is the original image and $h$ denotes the compared image.

For all examples in this section, the image is first scaled to have values in $[0,1]$; after denoising, it is scaled back for the 8-bit display. The constant components of the timestep size is set 1.78 for all cases, i.e., $\Delta t=1.78$. For the ITV-based models (ITV and M-ITV), the parameter $\lambda$ is set a constant in $[0.4,0.7]$ depending on the image under being processed. On the other hand, the ABO-based models select $\beta=\beta(\mathrm{x}, t)$ dynamically utilizing a given initial constant $\beta=0.4^{[9]}$. For MONTE-incorporated models (M-ITV and M-ABO), we set $\eta=1000$ and $\gamma$ is determined in each time level such that the arithmetic average of $F(s)$ becomes 1 over the whole domain:

$$
\eta=1000, \gamma=N_{x} N_{y}\left(\sum_{i=1}^{N_{x}} \sum_{j=1}^{N_{y}} \frac{1}{1+\eta\left|s_{i j}\right|}\right)^{-1}
$$

where $N_{x}$ and $N_{y}$ denote the number of pixels in

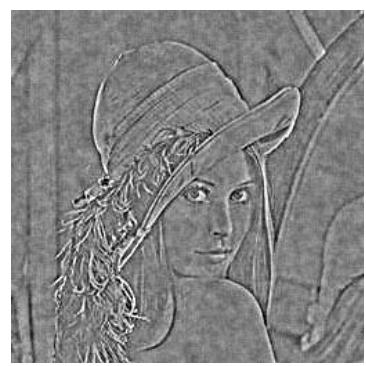

(a)

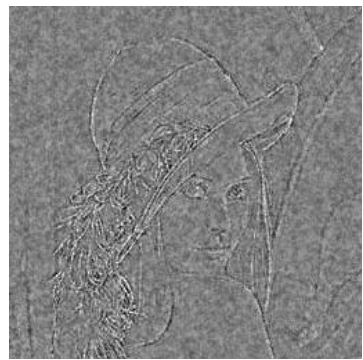

(c)

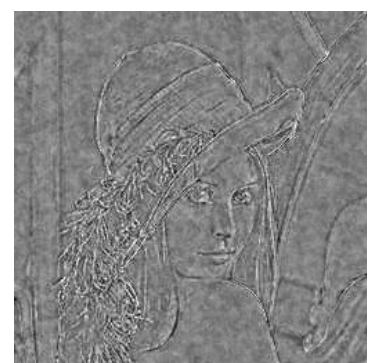

(b)

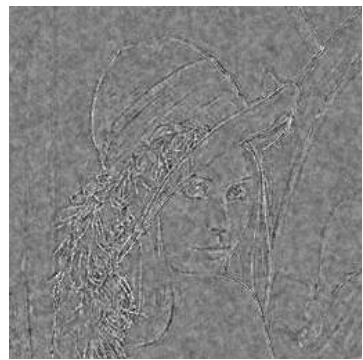

(d)
Fig. 4. Lenna: Magnified residuals $3^{*}(g-u)+128$, corresponding to the restored images in Figure 3 (in the same order)

the image in the horizontal and vertical directions, respectively. (The average of diffusion magnitude $|s|$ is ranged between 0.01 and 0.2 for typical real images.)

Figure 3 depicts restored images from the noisy image in Figure 2(b) by the four models. As one can see from Figure 3(a), the ITV model introduces a large numerical dissipation for the restored image to be blurry. The ABO and M-ITV models lose less fine details in their resulting images, while the $\mathrm{M}-\mathrm{ABO}$ model shows the best restored image as in Figure 3(d). See Table 1 below for a PSNR comparison.

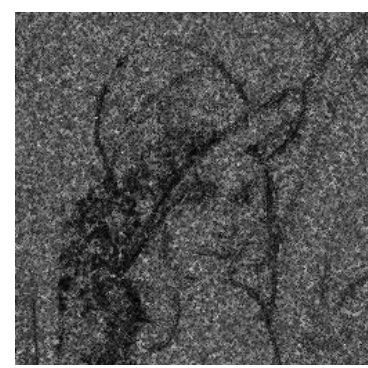

(a)

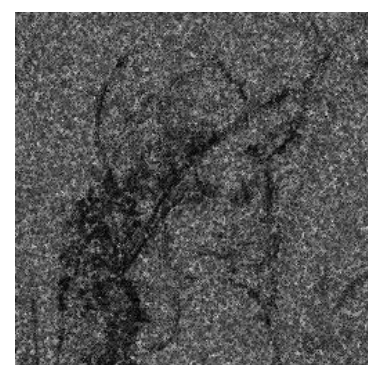

(b)
Fig. 5. The time surfaces on which the restored images are obtained for (a) M-ITV and (b) M-ABO. 


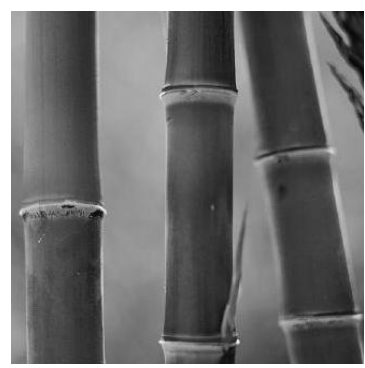

(a)

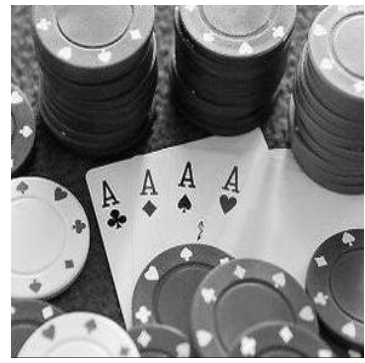

(c)

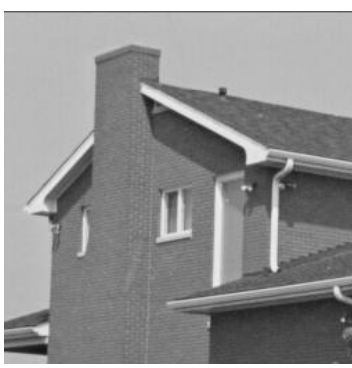

(b)

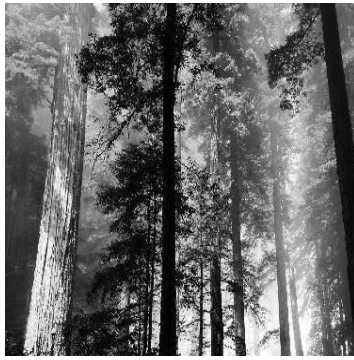

(d)
Fig. 6. Images utilized for a PSNR analysis: (a) Bamboo, (b) House, (c) Poker Chips, and (d) Forest.

In Figure 4, we present magnified residuals $3^{*}(g-u)+128$, for a quantitative comparison of the restored images in Figure 3, where $g$ is the original image in Figure 2(a) and $u$ denotes each of the restored images in Figure 3. As one can see from Figure 4(c) and Figure 4(d), the MONTE-incorporated models have produced significantly reduced residuals. Although some texture components of the image are shown in the magnified residuals, they are hardly noticeable in the (shifted) original residual $g-u+128$, in particular, for the M-ABO model.

Figure 5 contains the time surfaces for the MONTE-incorporated models on which the restored images are obtained. They show lower values on regions where the image shows larger values in the diffusion magnitude, as we have expected from the analysis in Section 3. It is clear to see that such a strategy of nonflat time evolution makes the net diffusion equalized over a wide range of image frequency components, which results in a significant reduction in numerical dissipation near fine structures.

From Figures 2-5, we have observed that denoising algorithms can significantly reduce numerical dissipation and then satisfactorily preserve fine structures, when they incorporate the MONTE with an ND function $s F(s)$ appropriately selected. Note that the ND function considered in this article ((17) and (19)) is concerned with diffusion equalization. The MONTE (along with the ND function) has been verified similarly effective for all images we have tested.

In Table 1, we present a PSNR analysis for the Lenna image considered in Figures 2-5 and a set of selected images as shown in Figure 6. The integers in parentheses denote the number of iterations in which the model can restore its best image (measured in the PSNR). As shown in the table, the

Table 1. PSNR analysis

\begin{tabular}{|l|c|c|c|c|c|}
\hline & $f$ & ITV & ABO & M-ITV & M-ABO \\
\hline Lenna & 24.8 & $28.0(2)$ & $30.9(10)$ & $31.0(2)$ & $31.3(3)$ \\
\hline Bamboo & 21.9 & $31.2(7)$ & $32.3(5)$ & $32.2(3)$ & $32.4(3)$ \\
\hline House & 19.9 & $28.8(7)$ & $29.6(4)$ & $29.8(4)$ & $29.8(3)$ \\
\hline Poker Chips & 19.1 & $24.2(4)$ & $26.2(10)$ & $26.1(3)$ & $26.4(3)$ \\
\hline Forest & 22.2 & $19.5(2)$ & $22.3(20)$ & $22.5(2)$ & $22.6(2)$ \\
\hline
\end{tabular}

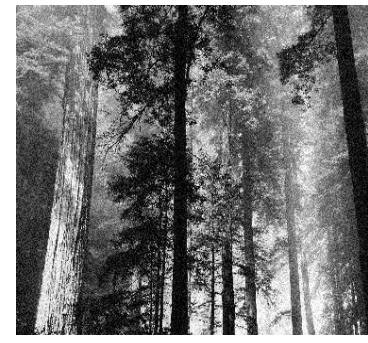

(a)

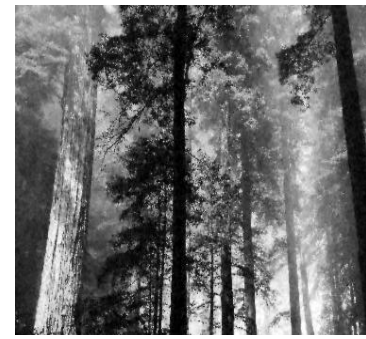

(c)

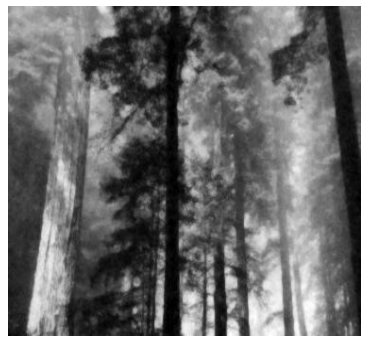

(b)

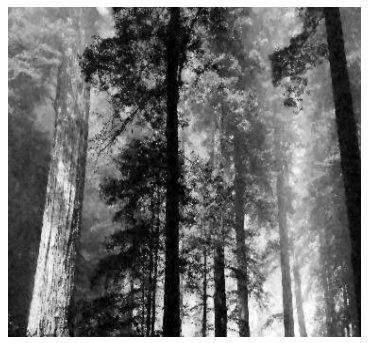

(d)
Fig. 7. Forest: The noisy image (a) and restored images by (b) the ITV model, (c) the M-ITV, and (d) the M-ABO. 
MONTE-incorporated models result in better PSNRs than the non-MONTE models, while the ABO-based models perform over the ITV-based models. The M-ABO model yields the highest PSNR and the best visual restored images, for all tested images including ones not presented in this article.

It should be noticed that the MONTE improves both efficiency and reliability. As shown in Table 1, the M-ITV and M-ABO models require 2-4 M-CN-ADI iterations for the best PSNRs. The ITV model can also restore its best images in two iterations for Lenna and Forest. However, these cases cannot be considered as a proof for a superior efficiency of the ITV model, because its restored images are unsatisfactory as indicated by the PSNRs that are much lower than those of other models. These rather imply that the ITV model is quick to remove high-frequency components of the image, including the noise and fine structures as well. On the other hand, for most tested images, the M-CN-ADI algorithm (18) reaches in 2-4 iterations the highest PSNR which is not only adequate but also larger than the ones obtainable with non-MONTE models.

Figure 7 contains the noisy image and three restored images for the Forest image considered in Table 1.

As one can see from Figure 7(b), the ITV model has destroyed fine structures in two CN-ADI iterations, while it is suppressing the noise; the restored image is too blurry to be tolerable.

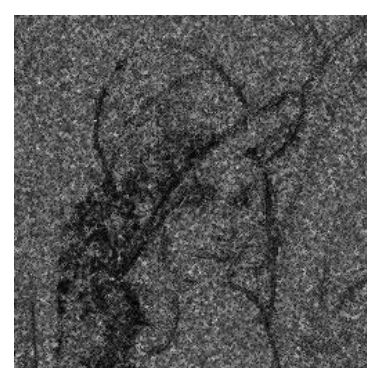

(a)

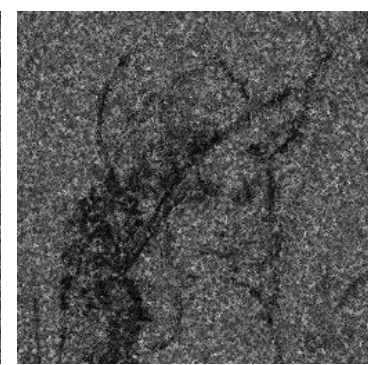

(b)
Fig. 8. The performance of the M-Heat. (a) The original image, (b) a noisy image having PSNR $=21.7$, and (c) the restored image by the M-Heat, having PSNR=31.8.

However, the MONTE-incorporated models
(M-ITV and $\mathrm{M}$-ABO) can restore comparable images just in two M-CN-ADI iterations, preserving satisfactorily fine structures of such a texture-enriched image.

In particular, the M-ABO model could recover great details of the image, as easily observed from a comparison between Figure 7(d) and the original Forest image in Figure 6(d). Fine structures in texture images are not only hard to preserve but also bringing up important and challenging problems in image restoration.

In order to further verify characteristics of the MONTE, we finally incorporate it with one of the worst restoration models, the linear homogeneous heat equation (the model (7) with $p=2$ ), and see how effectively the resulting model can restore fine structures. We will call the resulting model the M-Heat.

Figure 8 presents the performance of the M-Heat. For simplicity, we select a piecewise constant synthetic image as in Figure 8(a). The noisy image in Figure 8(b) is obtained by adding a Gaussian noise of $\mathrm{PSNR}=21.7$. The $\mathrm{M}$-Heat model requires three $\mathrm{M}-\mathrm{CN}-\mathrm{ADI}$ iterations to produce the restored image in Figure 8(c) of which PSNR=31.8.

The restored image shows relatively clear edges, although the background model is the heat equation representing heat transfer in homogeneous isotropic media. When the ITV model (without the MONTE) is applied for the noisy image in Figure 8(b), its best restored image turns out to have PSNR=30.4, which is worse than that of M-Heat.

The above example reveals a distinctive property of the MONTE: the preservation of fine structures through diffusion equalization.

\section{CONCLUSIONS}

Various PDE-based models have been studied to answer fundamental questions in image restoration and improve traditional restoration methods. However, the mathematical models show a common drawback: loss of fine structure. Thus it is very interesting and challenging to develop new strategies for PDE-based restoration models to enhance the 
preservation capability of fine structures such as edges and textures. This article has first analyzed the source of the drawback: most PDE-based models impose an excessive numerical dissipation near fine structures due to a larger diffusion magnitude. In order to suppress the undesirable dissipation, we have introduced the so-called method of nonflat time evolution (MONTE) that can equalize the net diffusion for various PDE-based models, by selecting the timestep size depending on local diffusion magnitudes. It has been numerically verified that the MONTE is efficient and reliable for both synthetic and real images. The new technique has shown attractive characteristics such as: (a) significant reduction of numerical dissipation, (b) successful preservation of fine structures, (c) convenient applicability to diverse PDE-based restoration models, and (d) improved efficiency. It is applicable to color image denoising, with similar efficiency and reliability for various representations and transformations of color images.

\section{ACKNOWLEDGMENT}

Kim's work is supported in part by the NSF grant DMS-1228337. The authors are grateful for the constructive and helpful suggestions from three anonymous referees.

\section{REFERENCES}

[1] WR.C. Gonzalez and R.E. Woods, Digital Image Processing, 2nd Ed. Prentice-Hall, Inc., Upper Saddle River, New Jersey, 2002.

[2] Y. Meyer, Oscillating Patterns in Image Processing and Nonlinear Evolution Equations, volume 22 of University Lecture Series, American Mathematical Society, Providence, Rhode Island, 2001.

[3] S.K. Mitra and G.L. Sicuranza, Nonlinear Image Processing. Academic Press, San Diego, San Francisco, New York, Boston, London, Sydney, Tokyo, 2001.

[4] G. Sapiro, Geometric partial differential equations and image analysis, Cambridge
University Press, Cambridge, 2001.

[5] P. Perona and J. Malik, "Scale-space and edge detection using anisotropic diffusion", IEEE Trans. on Pattern Anal. Mach. Intell., vol. 12, pp. 629-639, Jul. 1990.

[6] A. Marquina and S. Osher, "Explicit algorithms for a new time dependent model based on level set motion for nonlinear deblurring and noise removal". SIAM J. Sci. Comput., vol. 22, pp. 387-405, Aug. 2000.

[7] L. Rudin, S. Osher and E. Fatemi, "Nonlinear total variation based noise removal algorithms", Physica D, vol. 60, pp. 259-268, Nov. 1992.

[8] T. F. Chan and J. Shen, "Variational restoration of nonflat image features: Models and algorithms", SIAM Journal of Applied Mathematics, vol. 61, no. 4, pp. 1338-1361, Jul. 2006.

[9] S. Kim, "PDE-based image restoration: A hybrid model and color image denoising". IEEE Trans. Image Processing, vol. 15, no. 5, 1163-1170, May 2006.

[10] R. Kimmel and N. Sochen, "Orientation diffusion or how to comb a porcupine?", Special issue on PDEs in Image Processing, Computer Vision, and Computer Graphics, J. Visual Comm. Image Representation, vol. 13, pp. 238-248, Mar.-Jun. 2002.

[11] L. Alvarez, P.L. Lions and M. Morel, "Image selective smoothing and edge detection by nonlinear diffusion II", SIAM J. Numer. Anal., vol. 29, pp. 845-866, Jun. 1992.

[12] T. Chan, S. Osher, and J. Shen, "The digital TV filter and nonlinear denoising", IEEE Trans. Image Process., vol. 10, no. 2, pp. 231-241, Feb. 2001.

[13] Y.-L. You, W. Xu, A. Tannenbaum and M. Kaveh, "Behavioral analysis of anisotropic diffusion in image processing". IEEE Trans. Image Process., vol. 5, pp. 1539-1553, Nov. 1996.

[14] G. Aubert and P. Kornprobst, Mathematical Problems in Image Processing. Number 147 
in Applied Mathematics Sciences. Springer-Verlag, New York, 2002.

[15] S. Osher and R. Fedkiw, Level Set Methods and Dynamic Implicit Surfaces. Springer-Verlag, New York, 2003.

[16] S. Osher, M. Burger, D. Goldfarb, J. Xu, and W. Yin, Using geometry and iterated refinement for inverse problems (1): Total variation based image restoration. CAM Report \#04-13, Department of Mathematics, UCLA, LA, CA 90095, 2004.

[17] Y. Cha and S. Kim, "Edge-forming methods for color image zooming". IEEE Trans. Image Process., vol. 15, no. 8, pp. 2315-2323, Aug. 2006.

[18] Y. Cha and S. Kim, "Edge-forming methods for image zooming". J. Math. Imaging and Vis., vol. 25, no. 3, pp. 353-364, Oct. 2006.

[19] Y. Cha and S. Kim, "PDE-based image interpolators". Journal of Korea Information and Communication Society, vol. 35, no. 12, pp. 1010-1019, Dec. 2010.

[20] R. Weinstock, Calculus of Variations, Dover Publications, Inc., New York, 1974.

[21] J. Douglas, Jr. and J. E. Gunn, "A general formulation of alternating direction methods Part I. Parabolic and hyperbolic problems", Numer. Math., vol. 6, pp. 428-453, Dec. 1964.

[22] J. Douglas, Jr. and S. Kim, "Improved accuracy for locally one-dimensional methods for parabolic equations", Mathematical Models and Methods in Applied Sciences, vol. 11, no. 9, pp. 1563-1579, Dec. 2001.

[23] A.-L. Barabasi and H.E. Stanley, Fractal Concepts in Surface Growth, Cambridge University Press, New York, 1995.

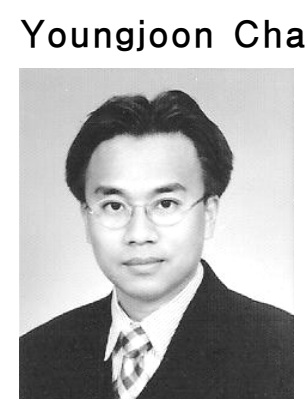

Professor, Department of Applied Mathematics, Sejong University 1988, B.S., Department of Mathematics, Seoul National University

1990, M.S., Department of Mathematics, Seoul National

University

1997, Ph.D., Department of Mathematics, Purdue University

$<$ Research Interests> Image Processing \& Computer Vision, Epidemiology, Wave Simulation

\section{Seongjai Kim}

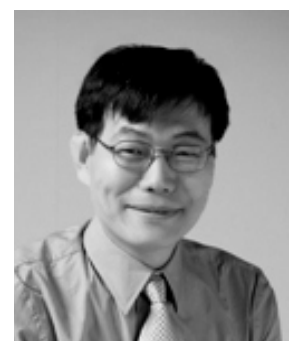

Associate Professor, Department of Mathematics and Statistics, Mississippi State University

1988, B.S., Department of Mathematics, Seoul National University.

1990, M.S., Department of Mathematics, Seoul National University.

1995, Ph.D., Department of Mathematics, Purdue University.

<Research Interests> Image Processing \& Computer Vision, Computational seismology, Wave Simulation 